\title{
Breast Cancer Therapeutic Procedure
}

National Cancer Institute

\section{Source}

National Cancer Institute. Breast Cancer Therapeutic Procedure. NCI Thesaurus. Code C15774.

Medical or surgical interventions to eradicate breast tumors and/or their metastases. 